\title{
Correction to: Late lumen enlargement after drug-coated balloon angioplasty for denovo coronary artery disease
}

\author{
Takayuki Onishi $^{1}$ (1) $\cdot$ Yuko Onishi $^{1} \cdot$ Isshi Kobayashi ${ }^{1} \cdot$ Yasuhiro Sato $^{1}$
}

Published online: 17 September 2020

(c) Japanese Association of Cardiovascular Intervention and Therapeutics 2020

\section{Correction to:}

Cardiovascular Intervention and Therapeutics (2020)

https://doi.org/10.1007/s12928-020-00690-2

In the original publication of the article, Table 3 was published incorrectly. The correct Table 3 is given in this Correction [1].

The original article has been corrected.

\section{Reference}

1. Onishi T, Onishi Y, Kobayashi I, Sato Y. Late lumen enlargement after drug-coated balloon angioplasty for denovo coronary artery disease. Cardiovascular Intervention and Therapeutics. 2020. https ://doi.org/10.1007/s12928-020-00690-2.

Publisher's Note Springer Nature remains neutral with regard to jurisdictional claims in published maps and institutional affiliations.

The original article can be found online at https://doi.org/10.1007/ s12928-020-00690-2.

Takayuki Onishi

takayuki.onishi@yahoo.com

1 Department of Cardiology, Hiratsuka Kyosai Hospital, 9-11

Oiwake, Hiratsuka, Kanagawa, Japan 
Table 3 Results from angiography

\begin{tabular}{|c|c|c|c|c|c|c|c|c|}
\hline \multirow{2}{*}{ Lesion, $n$} & & \multicolumn{2}{|l|}{ Total } & \multicolumn{2}{|l|}{ LLE } & \multicolumn{2}{|c|}{ No LLE } & \multirow[t]{2}{*}{$p$} \\
\hline & & 196 & & 109 & & 87 & & \\
\hline \multicolumn{9}{|l|}{ Preprocedure } \\
\hline $\mathrm{RVD}, \mathrm{mm}$ & & 2.07 & \pm 0.66 & 1.96 & \pm 0.55 & 2.20 & \pm 0.76 & 0.090 \\
\hline MLD, mm & & 0.86 & \pm 0.44 & 0.82 & \pm 0.44 & 0.91 & \pm 0.44 & 0.212 \\
\hline $\mathrm{DS}, \%$ & & 58.45 & \pm 19.31 & 58.48 & \pm 20.05 & 58.42 & \pm 18.46 & 0.408 \\
\hline Lesion length, mm & & 11.23 & \pm 6.07 & 11.03 & \pm 5.80 & 11.46 & \pm 6.39 & 0.721 \\
\hline \multicolumn{9}{|l|}{ Postprocedure } \\
\hline $\mathrm{RVD}, \mathrm{mm}$ & & 2.16 & \pm 0.57 & 2.03 & \pm 0.49 & 2.32 & \pm 0.64 & 0.002 \\
\hline MLD, mm & & 1.49 & \pm 0.42 & 1.37 & \pm 0.34 & 1.64 & \pm 0.47 & $<0.001$ \\
\hline DS, $\%$ & & 29.75 & \pm 14.59 & 31.18 & \pm 14.04 & 27.96 & \pm 15.14 & 0.065 \\
\hline Acute lumen gain, mm & & 0.64 & \pm 0.45 & 0.56 & \pm 0.43 & 0.74 & \pm 0.45 & 0.001 \\
\hline \multicolumn{9}{|l|}{ TIMI flow grade } \\
\hline 3 & & 189 & $(96 \%)$ & 106 & $(97 \%)$ & 83 & $(95 \%)$ & 0.476 \\
\hline 2 & & 5 & $(3 \%)$ & 3 & $(3 \%)$ & 2 & $(2 \%)$ & \\
\hline 1 & & 2 & $(1 \%)$ & 0 & $(0 \%)$ & 2 & $(2 \%)$ & \\
\hline \multicolumn{9}{|l|}{ Residual dissection } \\
\hline \multicolumn{9}{|l|}{ NHLBI classification } \\
\hline $\mathrm{A}$ & & 28 & $(14 \%)$ & 16 & $(15 \%)$ & 12 & $(14 \%)$ & 0.099 \\
\hline B & & 24 & $(12 \%)$ & 18 & $(17 \%)$ & 6 & $(7 \%)$ & \\
\hline $\mathrm{C}$ & & 15 & $(8 \%)$ & 9 & $(8 \%)$ & 6 & $(7 \%)$ & \\
\hline $\mathrm{D}$ & & 3 & $(2 \%)$ & 1 & $(1 \%)$ & 2 & $(2 \%)$ & \\
\hline $\mathrm{E}$ & & 1 & $(1 \%)$ & 1 & $(1 \%)$ & 0 & $(0 \%)$ & \\
\hline $\mathrm{F}$ & & 0 & $(0 \%)$ & 0 & $(0 \%)$ & 0 & $(0 \%)$ & \\
\hline \multicolumn{9}{|l|}{ Angiographic follow-up } \\
\hline Duration, months & & 7.2 & \pm 2.5 & 7.3 & \pm 2.6 & 7.2 & \pm 2.3 & 0.908 \\
\hline $\mathrm{RVD}, \mathrm{mm}$ & & 2.20 & \pm 0.65 & 2.19 & \pm 0.50 & 2.23 & \pm 0.82 & 0.110 \\
\hline MLD, mm & & 1.46 & \pm 0.48 & 1.63 & \pm 0.37 & 1.25 & \pm 0.51 & $<0.001$ \\
\hline DS, $\%$ & & 31.97 & \pm 19.10 & 24.64 & \pm 11.36 & 41.15 & \pm 22.62 & $<0.001$ \\
\hline Late lumen loss, mm & & 0.03 & \pm 0.43 & -0.26 & \pm 0.20 & 0.39 & \pm 0.37 & $<0.001$ \\
\hline Binary restenosis & & 42 & $(21 \%)$ & 6 & $(6 \%)$ & 36 & $(41 \%)$ & $<0.001$ \\
\hline \multicolumn{9}{|c|}{ Binary restenosis according to the ACC/AHA lesion type } \\
\hline A & $(n=22)$ & 1 & $(5 \%)$ & 0 & $(0 \%)$ & 1 & $(17 \%)$ & 0.273 \\
\hline B1 & $(n=59)$ & 10 & $(17 \%)$ & 2 & $(5 \%)$ & 8 & $(38 \%)$ & 0.002 \\
\hline B2 & $(n=80)$ & 19 & $(24 \%)$ & 3 & $(7 \%)$ & 16 & $(41 \%)$ & $<0.001$ \\
\hline $\mathrm{C}$ & $(n=35)$ & 12 & $(34 \%)$ & 1 & $(7 \%)$ & 11 & $(52 \%)$ & 0.006 \\
\hline
\end{tabular}

Data are presented as numbers $(\%)$ or means \pm standard deviations

ACC American College of Cardiology, AHA American Heart Association, DS diameter stenosis, LLE late lumen enlargement, NHLBI National Heart, Lung, and Blood Institute, $M L D$ minimal lumen diameter, $R V D$ reference vessel diameter, TIMI thrombolysis in myocardial infarction 